\title{
Emphysematous cystitis: An atypical multi-organism presentation
}

\author{
Derek Bos, ${ }^{*}$ Premal Patal, MD; ${ }^{\dagger}$ Sonia DiTullio, $M D^{\S}$
}

*Northern Ontario School of Medicine, Greater Sudbury, ON; †Department of Urological Sciences, University of Manitoba, Winnipeg, MB; §Department of Family Medicine, Timmins and District Hospital, Timmins, ON

Cite as: Can Urol Assoc J 2014;8(3-4):e210-2. http://dx.doi.org/10.5489/cuaj.1259 Published online March 11, 2014.

\section{Abstract}

An 84-year-old diabetic women receiving in-hospital stroke management began suffering from vague lower abdominal pain, increased urge incontinence and gross hematuria. Physical examination revealed suprapubic tenderness and a tympanic bladder. A computed tomography confirmed a distended bladder with gas foci delineating the bladder wall. Microbiology revealed an atypical multi-organism presentation of Klebsiella pneumoniae and Enterococcus species. Emphysematous cystitis is characterized by air within the bladder wall often found in elderly diabetic females. Predominant organisms include Escherichia coli and Klebsiella pneumonia. Prompt treatment is essential in this potentially fatal condition.

\section{Introduction}

Emphysematous cystitis (EC) is characterized by air within the bladder wall and lumen caused by fermentation of an underlying bacterial infection. The disease is often found in elderly female diabetics. Etiology is multifactorial and pathogenesis is poorly understood. Clinical presentation varies vastly; many cases are found incidentally on abdominal imaging. Emphysematous cystitis is quite rare. The rapid progression of the condition requires prompt treatment to ensure a favourable outcome. This case report and subsequent review of literature highlight the important aspects of this disease focusing on clinical presentation, diagnosis and treatment.

\section{Case report}

An 84-year-old female with significant medical comorbidities, receiving in-hospital stroke management, began complaining of vague lower abdominal pain. The pain was described as intermittent, and without radiation. Her urge incontinence worsened with complaints of gross hematuria.
She denied increased frequency and dysuria. There were no complaints of nausea, vomiting, fever, chills or flank pain. She did not have diarrhea, melena or frank blood in her stools.

Abdominal exam revealed mild suprapubic tenderness. The bladder was palpable at the level of the umbilicus and was tympanic to percussion. No other abnormalities were noted. Laboratory evaluation revealed a normal complete blood count, a baseline creatinine of $64 \mu \mathrm{mol} / \mathrm{L}$ and a hemoglobin A1C of $6.6 \%$.

Urinalysis showed trace ketones, a mildly elevated leukocyte esterase, negative nitrites, and microscopic hematuria.

An abdominal ultrasound was initially performed demonstrating a complex structure in the mid-pelvis, measuring about $12 \times 8 \mathrm{~cm}$, described as homogenous with increased echogenicity (Fig. 1). A computed tomography (CT) scan of the pelvis was subsequently performed, revealing a distended bladder measuring $10 \times 9 \mathrm{~cm}$ with extensive peripheral air in its inner margin and a significant amount of gas anteriorly compatible with emphysematous cystitis (Fig. 2, Fig. 3).

Intravenous (IV) ceftriaxone was started empirically and a 16-Fr two-way Foley catheter was inserted for bladder draining and urine cultures. Blood cultures were unremarkable. The urine culture initially grew Klebsiella pneumoniae. About 24 hours later a second organism, Enterococcus species, was isolated. Antibiotic treatment was adjusted to a 10-day course of IV cefazolin and oral nitrofurantoin based on organism sensitivities and minimum inhibitory concentration values.

To rule out emphysematous pyelonephritis and perinephric abscess, a CT of the abdomen/pelvis was completed 48 hours into treatment. The CT revealed interval resolution of gas tracking within the bladder wall with mild residual bladder wall thickening and no evidence of any renal involvement.

Following this 10-day course of antibiotic therapy, a repeat urine culture was completed. The cultures showed no growth. At that time her Foley catheter was removed. About 1 week later, the patient went into urinary retention with post-void residual values as high as $500 \mathrm{~mL}$. Clean 


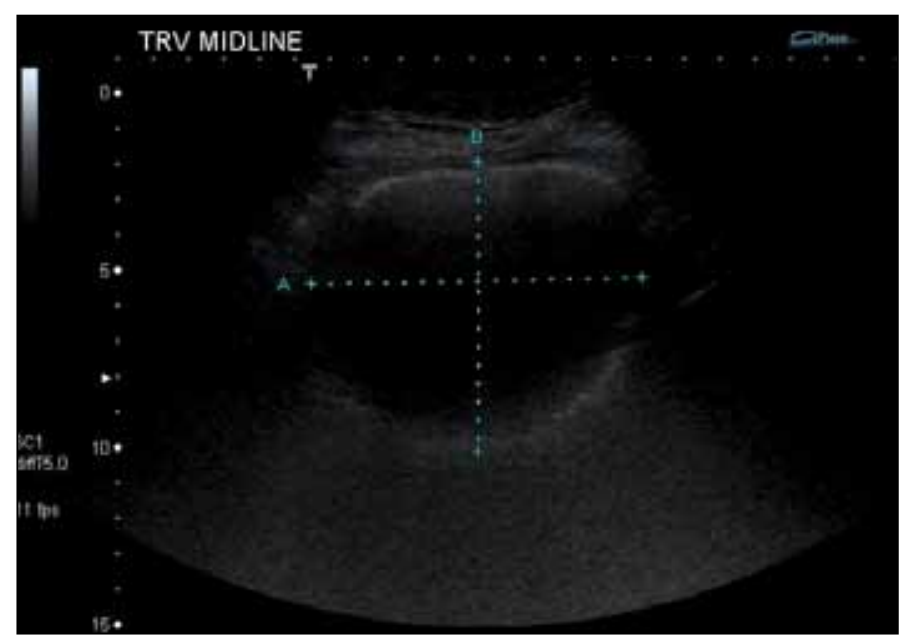

Fig. 1. An ultrasound image of the pelvis illustrating a mid-pelvis complex structure, measuring $12 \times 8 \mathrm{~cm}$ with a homogenous increased echogenicity.

intermittent catheterization was initiated to retrain the bladder for independent urination.

Cystoscopy was performed 4 weeks following initial presentation revealing inflammatory-induced hemorrhage into the submucosa indicative of resolved infection. The bladder wall was unremarkable with normal site and morphology of the ureteric orifices. Residual urine was sent for culture and sensitivity and was again positive for Klebsiella pneumoniae. Based on sensitivities, the patient was treated with a 7-day course of trimethoprim-sulfamethoxazole.

While hospitalized, the patient suffered from another cerebrovascular event significantly affecting her function. Due to the discomfort associated with routine intermittent catheterization over 4 weeks, the patient ultimately elected to have an indwelling Foley catheter inserted.

\section{Discussion}

Gas within the urinary tract was first described in 1671; it was further defined by Bailey in 1961 as "cystitis emphsematosa."1-3 EC is potentially fatal, as a result of a necrotizing infection that often begins in the bladder, rapidly ascending to the renal parenchyma. ${ }^{2,4}$ The etiology and pathophysiology of EC remain poorly understood. However, a multifactorial etiology of impaired host response with glucose or protein fermentation can explain the production of gas within affected tissues. It has been postulated that in diabetic patients high glucose concentrations within the urinary tract act as a favourable substrate for organisms to produce carbon dioxide and hydrogen through the natural fermentation process. Our patient had a history of well-controlled diabetes with an $\mathrm{A} 1 \mathrm{C}$ of $6.6 \%$. In non-diabetic patients high levels of urinary albumin, lactose, or tissue proteins can result in gas formation. Bacterial endotoxin release may also contribute to the inflammatory process by inducing paralysis

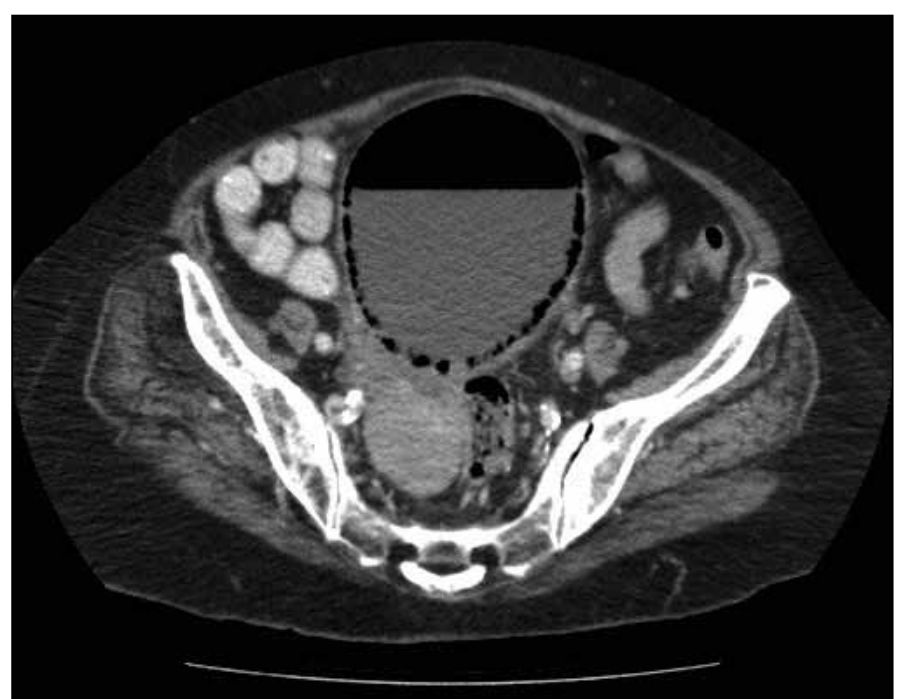

Fig. 2. Axial computed tomographic image through the lower pelvis, showing multiple punctate gas foci delineating the bladder wall. Intraluminal air also present anteriorly.

of the urinary tract, resulting in urinary stasis. ${ }^{5}$

The demographics of EC demonstrate a propensity for elderly diabetic women, as seen in this case report. Thomas and colleagues identified that two-thirds of cases of EC until 2006 were diabetic and $64 \%$ were female, with a median patient age of 66 years. ${ }^{6}$ Diabetes in EC patients could be higher, as roughly half of people with diabetes mellitus are undiagnosed. ${ }^{7}$ Diabetics are prone to complicated urinary tract infections (UTIs), including fungal infections, xanthogranulomatous pyelonephritis and gas-forming infections. ${ }^{8}$ Urinary stasis is another major risk factor. This case illustrates a model profile of a high-risk EC patient.

Prevailing organisms, as demonstrated by Thomas and colleagues, include Escherichia coli $(58 \%)$, followed by

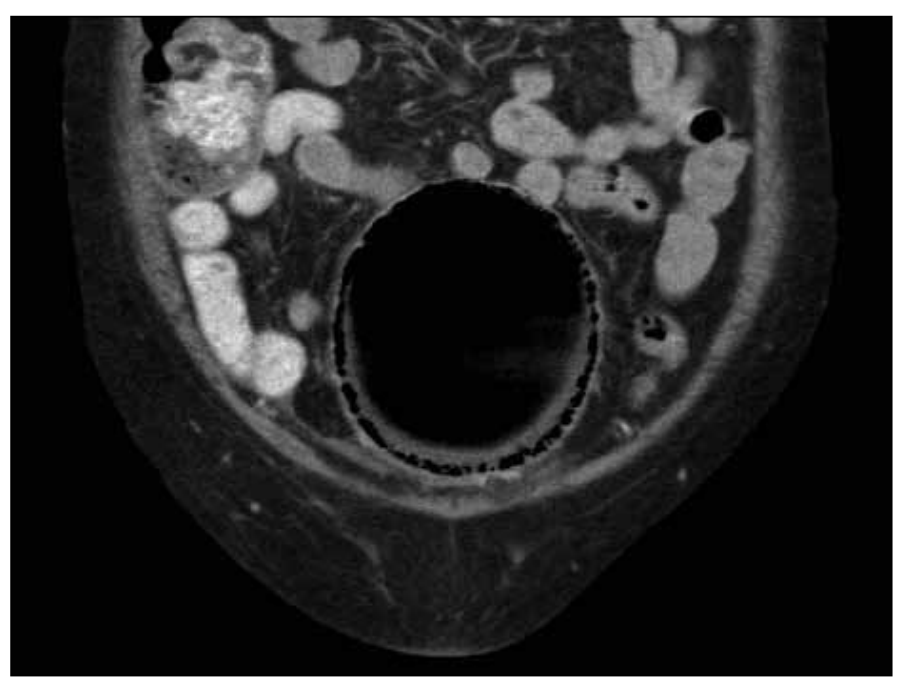

Fig. 3. Coronal computed tomographic image through the lower pelvis, demonstrating peripheral gas foci defining the bladder wall. Significant intraluminal gas noted. 
Bos et al.

Klebsiella pneumoniae (21\%), Clostridium species (7\%), and Enterobacter species (7\%). ${ }^{6}$ Most organisms are facultative anaerobic, while anaerobic isolate, multiple organisms, or no organisms are rarely found. ${ }^{9}$ This case exhibits an atypical multi-organism microbiology growing both Klebsiella pneumonia and Enterococcus species.

The clinical manifestations of EC are often non-specific. Many patients are asymptomatic or report only minor voiding difficulty or abdominal pain. Septic shock can be present at initial presentation, underlying the severity of this condition. In a study by Thomas and colleagues, about $7 \%$ of patients were asymptomatic. ${ }^{6}$ Gupper and colleagues reported that classic symptoms of UTI were present in only $50 \%$ of the cases. The most common symptom was abdominal pain, as seen in this case. ${ }^{10}$

The diagnosis of EC has seen a substantial increase recently due to a number of factors, including more awareness of the condition, higher volume of imaging, and a rise in the diabetic and elderly population. In contrast to typical UTIs, one cannot always rely on history and urinalysis for the diagnosis of EC. Urinalysis can be misleading as seen with the non-specific urine parameters noted in our case.

Suspicion is raised when elderly diabetic patients present with vague abdominal pain, incontinence, gross hematuria and a tympanic bladder on percussion. In our case, ultrasonography showed non-specific findings; therefore, a CT was used to diagnose the pathology. $\mathrm{CT}$ is the most accurate method of diagnosing EC, with a higher specificity than plain film. It can define the extent and severity of the disease and differentiate other pathology associated with the urinary tract, such as vesicocolic fistula, intra-abdominal abscess, or adjacent neoplastic disease. ${ }^{11-12}$ Abdominal ultrasonography is a less valuable imaging modality, as interpretation is often difficult. In our case, CT, leading to the eventual diagnosis, further characterized the undetermined mass described on ultrasonography. Cystoscopy alone is not diagnostic, but it can evaluate the presence of bladder outlet obstruction or confirm an enterovesical fistula. ${ }^{13}$

Management of EC often depends on its severity. Most often treatment consists of antibiotics, bladder drainage, glycemic control and treatment of any comorbid conditions. Empiric intravenous broad-spectrum antibiotic therapy is initially established. Gram-negative and anaerobic urinary pathogen antibiotic coverage is essential. Once sensitivities of isolated urinary pathogens are determined, antibiotics should be adjusted appropriately. Duration of treatment is not well-established. Grupper and colleagues found that a median length of 10 days is effective. ${ }^{10}$ In rare cases, when patients do not respond to conservative treatment, surgery may be required (cystectomy, surgical debridement).
As reported by Thomas and colleagues, the overall mortality rate is $7 \% .{ }^{6}$ Timely conservative treatment has resulted in a more favourable prognosis than initially predicted. However, when coexisting with another emphysematous infection of the urinary tract, the mortality rate can rise to $14 \%{ }^{6}$

\section{Conclusion}

The severity of EC underlies the importance in early detection. It is important to be aware of susceptible populations, including females, the elderly and diabetics. The non-specific and insidious presentation of EC can easily be missed based on history and physical examination. Imaging should be considered in a high-risk patient with undifferentiated abdominal pain of unknown origin with vague urinary symptoms. Timely diagnosis and treatment are essential in reducing morbidity and mortality of this rare disease.

Competing interests: Dr. Bos, Dr. Patal and Dr. DiTullio all declare no competing financial or personal interests.

This paper has been peer-reviewed.

\section{References}

1. Taussing AF. Pneumaturia with report of a case. Boston Med Surg J 1907;156:769-74. http://dx.doi.org/10.1056/NEJM190706131562403

2. Bailey H. Cystitis emphysematosa: 19 cases with intraluminal and interstitial collections of gas. Am J Roentgenol Radium Ther Nucl Med 1961;86:850-62.

3. Quint HJ, Drach GW, Rappaport WD, et al. Emphysematous cystitis: Review of the spectrum of disease. J Urol 1992;147:134-7.

4. Hawtrey CE, Williams JJ, Schmidt JD. Cystitis emphysematosa. Urology 1974;3:612-4. http://dx.doi.org/10.1016/S0090-4295(74)80259-1

5. Kapil RD. A case of complicated urinary tract infection: Klebsiella pneumonia emphysematous cystitis presenting as abdominal pain in the emergency department. West J Emerg Med 2008;9:171-3.

6. Thomas AA, Lane BR, Thomas AZ, et al. EC: A review of 135 cases. BJU Int 2007;100:17-20. http://dx.doi.org/10.1111/i.1464-410X.2007.06930.x

7. Ellenbogen PH, Talner LB. Uroradiology of diabetes mellitus. Urology 1976;8:413-9. http://dx.doi.org/10.1016/0090-4295(76)90505-7

8. Patterson JE, Andriole VT. Bacterial urinary tract infections in diabetes. Infect Dis Clin North Am 1997;11:735-50. http://dx.doi.org/10.1016/S0891-5520(05)70383-4

9. De Baets K, Joost, B, Coene L, et al. Emphysematous cystitis: Report of an atypical case. Case Rep Urol 2011;280426.

10. Gupper M, Kravtsov, Potasman I. Emphysematous cystitis: Illustrative case report and review of the literature. Medicine 2007;86:47-53. http://dx.doi.org/10.1097/MD.0b013e3180307c3a

11. Grayson DE, Abbott RM, Levy AD, et al. Emphysematous infections of the abdomen and pelvis: A pictorial review. Radiographics 2002;22:543-61. http://dx.doi.org/10.1148/radiographics.22.3.g02ma06543

12. Jarrett TW, Vaughan ED Jr. Accuracy of computerized tomography in diagnosis of colovesical fistula secondary to diverticular disease. J Urol 1995; 153:44-6. http://dx.doi.org/10.1097/00005392-199501000-00018

13. Thomas AA, Long RM, Creagh TA, et al. Emphysematous cystitis in an elderly patient with ulcerative colitits. Ir Med J 2006;99:56-7.

Correspondence: Mr. Derek Bos, Northern Ontario School of Medicine, Greater Sudbury, ON; debos@nosm.ca 